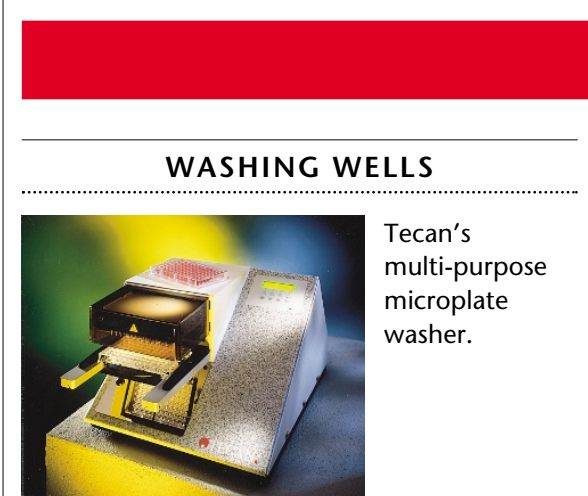

The specialized wash head of Tecan's 96 PW microplate washer allows the user to wash all 96 wells simultaneously, making the instrument particularly suited to highthroughput applications. Adjustable vacuum levels and dispensing speeds make it possible to switch between the fast washing speed needed for ELISAs and the gentle washing speed required for cell-based assays. Moreover, Tecan says that with the $96 \mathrm{PW}$, it is possible to achieve residual volumes as low as $2 \mu \mathrm{l}$ per well, ensuring reproducible wash results. The automated bubble sensor detects and alerts the user to air bubbles in the system. Also, the autorinse procedure allows uninterrupted use by preventing the crystallization of buffer in the wash head when the instrument is in stand-by mode. The instrument can be integrated into robotic liquid handling systems, if required.

Reader Service No. 94

Tel. (+41) (0) 55-254-8111

Fax $(+41)(0)$ 55-244-3883

TriContinent, a subsidiary of Hitachi Chemical, offers the new MultiWash Advantage microplate washer. It is intended for a wide range of applications from cell-based assays requiring gentle washing to more aggressive washing needed for ELISAs, including antigen washing. The company says the instrument's coaxial manifold design ensures consistency and flexibility. Users can define, program and save up to seven different wash sequences. Other features include a rinse-on-demand feature, control of the dispense speed for the entire dispense volume, crosswise aspiration and a bottom wash capability, and an optional 16-port manifold for increased throughput.

Reader Service No. 95

Tel. (+1) 530-273-8888

Fax (+1) 530-273-2586

\section{PRIMERS BY DESIGN}

CPG Ware primer design software from the Intergen Company is intended to sim-

\title{
ON THE MARKET
}

plify the design of primers used in methylation-specific PCR (MSP). As amplification of identical sequences that differ only in methylation status is the foundation of MSP, primer design can be critical to the success of the assay. The software can be accessed through the company's website (www.intergenco.com/cpgware). The program output consists of primer sequence sets that are capable of discriminating between methylated and unmethylated DNA, template DNA sequence with the primer locations identified, and relevant amplicon sizes. Subscriptions conferring unlimited access for 6 or 12 months are available. A demo copy is available on the website.

Reader Service No. 96

Tel. (+1) 800-431-4505

\section{KITS}

Ambion's paraffin block RNA isolation kit is designed for the fast and easy isolation of RNA from paraffin-embedded tissues. The company says that rare messages can be efficiently amplified from RNA purified using the kit. The 4-h procedure yields RT-PCR-ready RNA free of genomic DNA contamination.

Reader Service No. 97

Tel. (+1) 512-651-0200

Fax $(+1)$ 512-651-0201

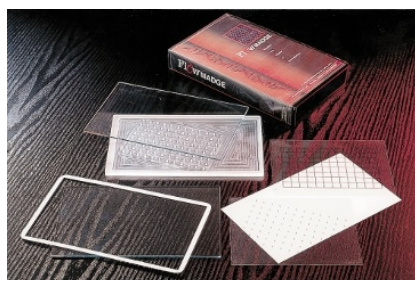

Flowmadge kit and software for high-throughput electrophoresis.

Flowgen's Flowmadge kit and AlphaMatch 1D software is designed for rapid, multisample electrophoresis in a traditional 96-well array. Typical sample types include PCR products, mini and microsatellites, gel-shift and protease assays, plus HPLC eluants. MADGE (microplate array diagonal gel electrophoresis) involves using a 96-well gel in exactly the same format as a standard 96-well microplate. This avoids complex sample recording and allows direct 8-, 12- or 96-well pipetting transfers. Additional marker lanes are standard for maximizing output and end-user convenience. Electrophoresis is carried out at an angle, the array being rotated through a small angle to maximize run lengths-typi- cally $26.5 \mathrm{~mm}$-and to maximize resolution. The Flowmadge unit is said to be compatible with standard electrophoresis tanks. Reader Service No. 98

Fax (+44) (0) 1530-419250

Email: support@flowgen.co.uk

\section{LIQUID DISPENSING DEVICES}

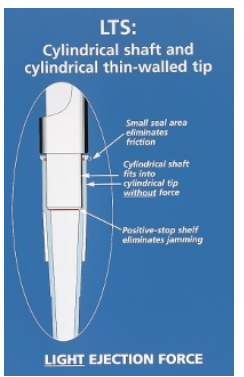

Rainin's ergonomic pipette and tip system.

The design of Rainin's new LTS LiteTouch tip ejection system is said to reduce tip ejection forces by up to $90 \%$, helping to reduce or eliminate repetitive strain injuries. The company now offers three new ergonomic pipettes that incorporate the LTS system: the Pipet-Lite with magnetic assist, the Pipet-Plus latch-mode manual pipettes and the EDP3 electronic pipette. At the heart of the design is a pipette shaft and tip that is cylindrical, rather than conical, and engineered to create a strong seal over a small, well-defined area. Moreover, LTS tips have a positive-stop 'shelf' that indicates when a proper seal has been made, thereby eliminating jamming.

Reader Service No. 99

Tel. (+1) 510-654-9142

Fax (+1) 510-652-1466

With Hamilton's Microlab 500C series of diluter/dispensers, you can use your own IBM-compatible computer to control the diluting and dispensing process. The Microlab 500C uses a precision syringe pump to provide a stated precision of $\pm 0.8 \%$ and a stated accuracy of $\pm 1.0 \%$ at $10 \%$ of the syringe volume. Other features include syringe speeds from 1 to $250 \mathrm{~s}$ per stroke and the ability to 'daisy chain' up to 16 units. Three different models are available: The single-syringe dispenser, the dual-syringe diluter and the dual-syringe dispenser.

Reader Service No. 100

Tel. (+1) 702-858-3000

Fax $(+1)$ 702-856-7259

For more details, fill in the reader service card at the back of the journal. 